Running head: EMOTIONS IN TECHNOLOGY-MEDIATED INTERACTIONS

\title{
Emotional Experiences in Technology-Mediated and In-Person Interactions: An Experience-Sampling Study
}

Kate Petrova* and Marc S. Schulz

Psychology Department, Bryn Mawr College, Bryn Mawr, PA, USA

* Correspondence concerning this article should be addressed to Kate Petrova, Psychology

Department, Stanford University, 450 Jane Stanford Way, Building 420, Stanford, CA 94305.

Email:kpetrova@stanford.edu. (D) https://orcid.org/0000-0003-1671-9768

This is an Accepted Manuscript of an article published in Cognition and Emotion, available online: https://www.tandfonline.com/doi/full/10.1080/02699931.2022.2043244. 


\begin{abstract}
As the ubiquity of technology-mediated communication grows, so does the number of questions about the costs and benefits of replacing in-person interactions with technology-mediated ones. In the present study, we used a daily diary design to examine how people's emotional experiences vary across in-person, video-, phone-, and text-mediated interactions in day-to-day life. We hypothesized that individuals would report less positive affect and more negative affect after less life-like interactions (where in-person is defined as the most life-like and text-mediated as the least life-like). In line with this hypothesis, the analysis of 527 unique interactions reported by 102 individuals (mean age $=19.3 ; 85.6 \%$ female) over the course of 7 days reveals that people feel lonelier, sadder, less affectionate, less supported, and less happy following less lifelike interactions. Additional analyses show that the links between life-like communication and momentary experiences are independent of properties of individual interactions such as interaction length and participants' overall evaluations of interaction quality. These findings provide initial evidence that there may be inherent properties of common technology-mediated communication tools that may lead to momentary changes in affective experiences and make social connection more challenging.
\end{abstract}

Keywords: social interactions, connection, loneliness, technology-mediated communication, affect 


\section{Emotional Experiences in Technology-Mediated and In-Person Interactions: An Experience-Sampling Study}

People spend most of their lives in the company of others. Though our proclivity for spending time with others may not have changed much since the early days of human civilization, how we interact has changed dramatically. From texting and videoconferencing to more "old-fashioned" in-person conversations, there are many ways for people in the $21^{\text {st }}$ century to connect. A critical question is, are technology-mediated moments of connection equivalent to their in-person counterparts? In the present study, we examine how affective experiences vary across several modes of technology-mediated and in-person interactions in day-to-day life.

Time and again, the advance of new technologies has spurred widespread concerns about the effects of technological innovations on the health and well-being of society (Orben, 2020). The advance of telecommunication technology in the $20^{\text {th }}$ and $21^{\text {st }}$ centuries has been no exception (Orben, 2020). An avalanche of research in recent decades has somewhat lessened fears of calamitous effects of technology on mental health (Panova \& Carbonell, 2018), but important questions remain. One domain of well-being that may be particularly influenced by replacing in-person interactions with technology-mediated ones is our need for connection.

Powerful moments of connection that are woven into the fabric of everyday life are fundamental to social and emotional well-being (e.g., Mehl et al., 2010; Sun et al., 2020). From eye-contact to affective synchrony, previous studies have identified a variety of features of inperson interactions that help people feel close and connected (see Hietanen, 2018; Lwi et al., 2019; Wood et al., 2020). What has received less empirical attention are the affective consequences of the fact that many of these features can be hard to recreate in digital 
environments. The experience of direct eye-contact is hard to replicate even in the most life-like ${ }^{1}$ forms of technology-mediated interactions, like videoconferencing. Similarly, limited availability of facial and vocal cues (e.g., smiling, tone of voice, etc.) in phone- and text-based interactions can make it particularly challenging to achieve affective synchrony.

The notion that less life-like forms of remote socializing make it more challenging to transmit social cues has been emphasized in influential theories such as the Social Presence Theory (Short et al., 1976) and the Media Richness Theory (Daft \& Lengel, 1986). The implications of reduced cue-transmissibility have been studied across a variety of domains (see review by Oh et al., 2018). The Social Presence and similar frameworks have also been invoked to inform research on close relationships and social functioning (e.g., Gooch \& Watts, 2015), but many theoretical and empirical questions about the impacts of technology-mediated communication (TMC) on emotional experiences and connection remain.

Despite strong theoretical reasons to believe that TMC could make social connection more challenging in some key ways, empirical support for this idea has been limited. For example, though recent studies have found that people evaluate in-person interactions as being more connecting compared to technology-mediated ones, the sizes of those associations have been small (e.g., Kushlev \& Heintzelman, 2018; Sherman et al., 2013). One possible explanation for these results is that certain properties of less life-like forms of TMC, like physical distance or the inability to achieve affective synchrony, may impose more subtle social and emotional costs that are relatively independent of individuals' explicit evaluations of connection quality. For example, the mere awareness of spatial distance between oneself and one's interaction partner in

\footnotetext{
${ }^{1}$ Throughout this paper, we use the term "life-likeness" to refer to the degree of similarity between different forms of technology-mediated communication and typical in-person interactions. Subtle features like eye contact, synchronicity, spatial proximity, and other such characteristics that may contribute to feelings of social connection but that are not necessarily related to media richness add up to higher life-likeness.
} 
an otherwise warm and positive interaction may increase perceptions of social distance (Won et al., 2018) and give rise to momentary feelings of loneliness. Similarly, the inability to pick up on emotional cues when turning to others for support in times of stress could render technologymediated support less effective and fail to dampen the intensity of negative emotions like anxiety or sadness (though evidence linking technology-mediated support to affective outcomes has been mixed: Holtzman et al., 2017, but see also Colasante et al., 2020).

TMC may also affect momentary experiences of positive emotions. Past research shows that sharing positive emotions with others amplifies their intensity (e.g., Morelli et al., 2015). This benefit of positive sharing can be partially attributed to listeners' active and enthusiastic responses to the shared content (Hovasapian \& Levine, 2018). Facial and vocal cues play an important role in conveying enthusiasm, which is why sharing positive emotions in less life-like interactions may not be as effective in boosting their intensity. Of course, it is possible that limited availability of emotional cues in some forms of TMC could also make upsetting and otherwise unpleasant social interactions (e.g., sharing or receiving bad news) feel less negative. In fact, there could even be systematic differences in the nature of interactions that take place across different media (e.g., some people may prefer to have difficult conversations via less lifelike media and to keep such conversations short). For these reasons, measuring and controlling for properties of individual interactions like emotional valence, perceived meaningfulness, and length is a critical step in clarifying the unique contributions of interaction life-likeness to affective experiences.

Though the momentary effects of TMC on affective experiences may be relatively small, as people move more and more of their daily interactions into the digital space, it is critical to consider the possibility that even small effects may add up over time and have longer-term 
effects on social and emotional well-being (see Kushlev et al., 2019; Verduyn et al., 2021). In recent years, studies have begun to use intensive longitudinal methods to better understand how TMC contributes to socio-emotional outcomes in real time (e.g., Colasante et al., 2020; Verduyn et al., 2021). Extending this important work by focusing on the momentary effects of TMC on emotional experiences at the more granular level (e.g., feelings of loneliness, happiness, or sadness rather than the more global perceptions of positivity) while controlling for individuals' evaluations of overall interaction quality is a critical research task. Here, we examine how people's experiences of positive and negative affect across six discrete emotion categories vary across in-person, video-, phone-, and text-mediated interactions across seven consecutive days. We predicted that individuals would report more positive affect and less negative affect after more life-like social interactions (where in-person is defined as the most life-like and texting as the least life-like).

\section{Method}

\section{Participants}

The sample consists of 106 participants ( $85.6 \%$ female) aged between 18 and 22 years $(M$ $=19.30, S D=1.14$ ) from a small liberal arts college in the North-eastern United States. The recruitment email was sent to the entire undergraduate student body, with the disclaimer that the study was only open to students currently living on campus. All participants were additionally asked to confirm that they were living on campus at the time informed consent was obtained. Participants did not receive any compensation for this study.

An a priori sample size estimation was carried out using the $p w r$ package in $\mathrm{R}$ (Champely et al., 2018) and showed that at least 193 observations would be required to detect a small-to-medium effect size $(f=.20)$ at a significance level of $\alpha$ (two-sided $)=.05$ with .80 
power. To adjust this estimate for clustering effects, we calculated a design effect (Muthén \& Satorra, 1995) for an intraclass correlation (ICC) of .25 and a cluster size (number of interactions reported by each participant) of 7 . Implementing the correction resulted in an estimate of at least 483 unique interactions required for detection of a small-to-medium effect size with .80 power.

\section{Procedure}

The data were collected in October 2020 as part of a larger initiative aimed at understanding the experiences of college students during the COVID-19 pandemic. The full protocol was approved by the Institutional Review Board at Bryn Mawr College and is available online at https://doi.org/10.17605/OSF.IO/F4BVW. Upon signing the informed consent form, participants completed a brief questionnaire in which they provided demographic information, answered questions about their experiences living on campus (e.g., number of roommates, involvement in clubs and other activities), and completed personality and loneliness inventories. The brief daily questionnaires were emailed to participants every day starting on the day after the initial questionnaire was submitted (Days 2-8). The daily questionnaires asked participants to think about their most recent social interaction and to answer a series of questions about the interaction, including communication mode (in-person, video, phone, or text), length of the interaction in minutes, perceived quality of the interaction (perceived positivity, meaningfulness, and connection), and whether or not the interaction was initiated by the participant. Participants were then asked to report their affective states across six discrete categories. The link to the daily questionnaire was sent to participants via email at random times each day between 9am and 9pm. Participants were instructed to fill out the questionnaire within 1 hour of receiving the email.

\section{Measures}

\section{Social Interactions and Subjective Evaluations of Interaction Quality}


During each daily assessment, participants answered six questions about their most recent interaction (defined as an in-person, phone, video, or text conversation lasting at least 2 minutes and involving back-and-forth communication) : (1) Did the interaction take place $(0=$ in-person $1=$ over video, $2=$ over phone, $3=$ over text $) ;(2)$ Did you initiate the interaction $?(0=$ No, $1=$ Yes); (3) To the best of your ability, estimate the duration of the interaction in minutes; (4) Overall, how positive was the interaction? (5) Overall, how connected did you feel to your interaction partner(s)? (6) Overall, how meaningful was the interaction to you? Questions 4-6 were rated on a 3 -point scale $(1=$ not at all, $2=$ somewhat, $3=$ very $)$. Questions 4,5 , and 6 were averaged together to create a total perceived interaction quality score (average Cronbach's $\alpha$ across the seven-day period $=.74)$.

\section{Momentary Affective Experiences}

Participants were presented with 6 triplets (adapted from Tamir et al., 2007):

lonely/distant/isolated, sad/depressed/down, anxious/worried/fearful, happy/pleased/contented, affectionate/loving/connected to others, and (developed for this study)

understood/supported/heard. Participants were asked to indicate the extent to which they felt each triplet at the time of assessment using a scale ranging from $1=$ not at all, to $4=$ somewhat, to $7=$ very much .

\section{Data analysis}

To examine the relationship between interaction mode and self-reported emotions at the within-person level, a series of multilevel structural equation models with random-intercepts ${ }^{2}$ were estimated in Mplus (Version 8.3, Muthén \& Muthén, 1998-2017). The associations between momentary emotion reports and modes of individual interactions were modeled at level 1

\footnotetext{
${ }^{2}$ Preliminary model building showed that there was no significant variation across participants in slopes (see Table $\mathrm{S} 1$ in the Supplementary Materials for additional details).
} 
(within-person). To decompose within- and between-person effects, we implemented a latent variance decomposition approach in Mplus (See p.1 of the Supplementary Materials for details). Days of the week (weekday $=0$, weekend = 1) and days of the study (ranging from 1 to 7 ) were controlled for in all analyses. Because there was little variation in participants' age and gender, the decision was made not to include age and gender as control variables in the main analyses. All Mplus code that was used to carry out the main analyses is available at https://doi.org/10.17605/OSF.IO/F4BVW.

\section{Results}

Data on 527 unique interactions were recorded across all participants over the course of 7 days. Of those interactions, $57.5 \%$ took place in-person, $26.2 \%$ took place over video, $4.9 \%$ took place over phone, and $11.4 \%$ took place over text. Out of the 106 participants who enrolled in the study, 102 (96\%) completed at least one daily assessment. The median number of completed assessments was 6. Of those who completed at least one daily assessments, 93 (91.2\%) participants completed at least two, and $41(40.2 \%)$ completed all seven. Preliminary analyses revealed that the number of completed assessments was not associated with any variables of interest. Full-information maximum likelihood (FIML) estimation was used in all multilevel analyses, which allowed us to use data from all 102 participants. Means, standard deviations, intraclass correlations, and bivariate within-person correlations for key study variables are presented in Table 1.

To examine the main hypothesis that individuals would report more positive affect and less negative affect following more life-like social interactions, we first modelled communication mode (ranging from $0=$ in-person to $3=$ text) as an ordinal predictor of affect ratings in a twolevel linear regression. Consistent with our expectations, we found that participants reported 
more negative affect and less positive affect following less life-like social interactions (Figure 1). Specifically, participants reported more loneliness, $\beta=.19,95 \%$ CI $[.09, .28], p<.001$, more sadness, $\beta=.17,95 \%$ CI [.08. .27], $p<.001$, and more anxiety, $\beta=.10,95 \%$ CI $[.006, .20], p=$ .04 following less life-like interactions. Participants also reported feeling less affectionate, $\beta=-$ $.21,95 \%$ CI $[-.31,-.11], p<.001$, less understood, $\beta=-.18,95 \%$ CI $[-.26,-.09], p<.001$, and less happy, $\beta=-.21,95 \%$ CI [-.30, -.11], $p<.001$, following less life-like interactions.

To account for potential systematic differences in the nature of social interactions that take place via different media, we expanded our original models (that used communication mode as an ordinal predictor of momentary affect) to include three additional control variables: (a) subjective evaluations of overall interaction quality (positivity, meaningfulness, and connection), (b) length (in minutes) of the interaction, and (c) whether or not the interaction was initiated by the participant. Consistent with the view that less life-like social interactions could make social connection more challenging, there was a negative correlation between life-like communication and perceived connection, though the size of that association was small, $r=-.18, p<.001$.

Overall, across the 7 days of the study, less life-like interactions tended to be shorter, $r=-.28, p$ $<.001$ and perceived as less positive, $r=-.29, p<.001$ as well as less meaningful, $r=-.21, p=$ .001. Controlling for these interaction properties at both levels, the links between interaction mode and self-reported emotions at the within-person level remained significant across all emotions except anxiety. The level-1 and level-2 coefficients derived from these models are presented in Table S2 in the Supplementary Materials.

\section{Discussion}

The human mind has been honed across millennia to navigate the social world, but adapting to the socio-digital terrain of the $21^{\text {st }}$ century raises new challenges. The current study 
focused on one such challenge and examined whether people's affective experiences in social interactions vary across in-person, video-, phone-, and text-based modes of communication. Results indicate that people feel happier, more affectionate, and more understood, as well as less lonely and less sad after engaging in social interactions that are more life-like (i.e., interactions that share more features - like eye-contact or synchrony - with typical in-person communication).

Our findings help bridge the literature on the emotional underpinnings of social connection (e.g., Lwi et al., 2019; Sun et al., 2020; Wood et al., 2020) with earlier work in the area of human-computer interaction (e.g., Short et al., 1976; Daft \& Lengel, 1986). Consistent with our expectations, the links between life-like communication and momentary affective experiences in the present study were of small to medium magnitude. We also found that participants reported feeling less connected to their interaction partners in less life-like social interactions. The size of this association was small, though highly similar to the effect sizes reported in previous studies of perceived connection in TMC (e.g., Kushlev \& Heintzelman, 2018). Importantly, the relationship between life-like communication and momentary affective experiences remained even when perceived connection and factors such as interaction length and perceived positivity were taken into account. These findings are broadly consistent with the idea that properties of less life-like forms of TMC (e.g., affective asynchrony, spatial distance, or limited availability of emotional cues) may influence affective experiences and interpersonal outcomes in subtle ways that are difficult to capture with questions that explicitly ask individuals to evaluate overall interaction quality. One alternative explanation for this pattern of results that is worth considering is that perceived interaction quality plays a causal role in mediating the 
effects of communication mode on affective experiences. Future studies with more temporally sensitive designs should explore this possibility.

Our findings add to the efforts of previous studies that employed within-person designs to track the same individuals across multiple interpersonal exchanges and document how their emotional responses depend on the mode of communication (e.g., Colasante et al., 2020;

Kushlev \& Heintzelman, 2018; Verduyn et al., 2021). Previous research shows that, on average, people who spend more time using technology to communicate report lower subjective wellbeing (e.g., Twenge et al., 2018). Our findings extend this important work by more clearly delineating the links between TMC and affective experiences at the level of discrete momentary emotions. Specifically, we show that people tend to feel lonelier, sadder, less affectionate and understood, as well as less happy (though not always more anxious) after they engage in less life-like interactions. These findings point to potential specificity in the effects of communication modes on discrete emotions. Understanding the sources and implications of such specificity as it relates to life-likeness is an important direction for future research. Within-individual differences in affective experiences across different forms of technology-mediated communication (video vs. phone; video vs. text; text vs. phone) should also be examined more closely in future studies.

The insights from this work also hold potentially important practical implications for efforts to enhance user experience with technology. The affective and social consequences of any given interaction are likely shaped by many factors, including individuals' motivations for engaging in TMC, their level of familiarity with specific communication tools, as well as the unique features of those tools. Building on the findings from the present work to understand how these factors interact could help design communication tools that augment the benefits and buffer against the potential negative effects of moving social interactions into the digital arena. 
Importantly, the findings from the present study should not be taken to imply that TMC is overall maladaptive. Understanding the longer-term consequences of remote interactions is challenging but critical. On the one hand, even small reductions in positive affect and the quality of connection may accrue over time, contributing to declines in social and emotional well-being. On the other hand, the increased frequency of social contact and the TMC-enabled ability to connect individuals who are far away could reduce loneliness and improve overall affective wellbeing over longer periods of time. Identifying the benefits of TMC (e.g., Valkenburg \& Peter, 2007) and weighing them against the potential downsides is a critical research goal. Future studies should also clarify whether engaging in TMC is associated with social and emotional advantages compared to not socializing at all (see Kushlev \& Heintzelman, 2018 for a related discussion).

A primary strength of the current research is the experience-sampling design that allows for a powerful and ecologically valid approach to examining the links between communication modes and affect in day-to-day life. There are also some limitations that need to be acknowledged.

The non-experimental design limits our understanding of the direction of influence between life-likeness of individual interactions and momentary affective experiences. Though there are theoretical and empirical reasons to believe that the degree of life-likeness of different communication modes may directly influence people's emotions, individuals' choice of an interaction medium may also be driven by their affective experiences (e.g., people may prefer less life-like modes of communication when they feel more negatively). Experimental studies that manipulate interaction mode directly would aid in establishing the direction(s) of influence. The within-person correlations in the present study are based on a limited number of 
observations per person (median $=6$ out of 7 ). In addition, the frequency of phone-based interactions in the present study was much lower than other modes of communication $(N=26$ out of 527). The lack of a measure of the average amount of time that had passed between reported interactions and survey completion limits our ability to draw nuanced conclusions about the time course of the affective sequelae of different types of interactions. Finally, though there are clear advantages to using a college-based sample consisting of young adults (e.g., high number of individuals engaging in TMC, shared environment), it may limit generalizability of current findings to older and more diverse populations. The fact that the study was conducted during the COVID-19 pandemic may similarly have implications for generalizability.

In conclusion, the findings from this study point to important ways in which momentary affective experiences differ across in-person, video-, phone, and text-mediated interactions and highlight the importance of understanding the power of these differences to affect long-term health and well-being.

\section{Data availability statement}

All materials and data that were used in this study as well as the full text of the manuscript (a preprint) are publicly available: https://doi.org/10.17605/OSF.IO/F4BVW.

\section{Disclosure of interest}

The authors report no conflict of interest. 


\section{References}

Champely, S., Ekstrom, C., Dalgaard, P., Gill, J., Weibelzahl, S., Anandkumar, A., ... \& De Rosario, M. H. (2018). Package 'pwr'. R package version, 1(2).

Colasante, T., Lin, L., De France, K., \& Hollenstein, T. (2020). Any time and place? Digital emotional support for digital natives. American Psychologist. https://doi.org/10.1037/amp0000708

Daft, R. L., \& Lengel, R. H. (1986). Organizational Information Requirements, Media Richness and Structural Design. Management Science, 32(5), 554-571. https://doi.org/10.1287/mnsc.32.5.554

Gooch, D., \& Watts, L. (2015). The Impact of Social Presence on Feelings of Closeness in Personal Relationships. Interacting with Computers, 27(6), 661-674. https://doi.org/10.1093/iwc/iwu020

Hietanen, J. K. (2018). Affective Eye Contact: An Integrative Review. Frontiers in Psychology, 9, 1587. https://doi.org/10.3389/fpsyg.2018.01587

Holtzman, S., DeClerck, D., Turcotte, K., Lisi, D., \& Woodworth, M. (2017). Emotional support during times of stress: Can text messaging compete with in-person interactions? Computers in Human Behavior, 71, 130-139. https://doi.org/10.1016/j.chb.2017.01.043

Hovasapian, A., \& Levine, L. J. (2018). Keeping the magic alive: Social sharing of positive life experiences sustains happiness. Cognition and Emotion, 32(8), 1559-1570. https://doi.org/10.1080/02699931.2017.1422697

Kushlev, K., Dwyer, R., \& Dunn, E. W. (2019). The Social Price of Constant Connectivity: Smartphones Impose Subtle Costs on Well-Being. Current Directions in Psychological Science, 28(4), 347-352. https://doi.org/10.1177/0963721419847200 
Kushlev, K., \& Heintzelman, S. J. (2018). Put the Phone Down: Testing a Complement-Interfere Model of Computer-Mediated Communication in the Context of Face-to-Face Interactions. Social Psychological and Personality Science, 9(6), 702-710. https://doi.org/10.1177/1948550617722199

Lwi, S. J., Haase, C. M., Shiota, M. N., Newton, S. L., \& Levenson, R. W. (2019). Responding to the emotions of others: Age differences in facial expressions and age-specific associations with relational connectedness. Emotion, 19(8), 1437-1449. https://doi.org/10.1037/emo0000534

Mehl, M. R., Vazire, S., Holleran, S. E., \& Clark, C. S. (2010). Eavesdropping on Happiness: Well-Being Is Related to Having Less Small Talk and More Substantive Conversations. Psychological Science, 21(4), 539-541. https://doi.org/10.1177/0956797610362675

Morelli, S. A., Lieberman, M. D., \& Zaki, J. (2015). The Emerging Study of Positive Empathy: Positive Empathy. Social and Personality Psychology Compass, 9(2), 57-68. https://doi.org/10.1111/spc3.12157

Muthén, L. K., \& Muthén, B. O. (1998-2017). Mplus User's Guide (8th ed.). Los Angeles: Muthén \& Muthén.

Muthen, B. O., \& Satorra, A. (1995). Complex sample data in structural equation modeling. Sociological Methodology, 25, 267. https://doi.org/10.2307/271070

Oh, C. S., Bailenson, J. N., \& Welch, G. F. (2018). A Systematic Review of Social Presence: Definition, Antecedents, and Implications. Frontiers in Robotics and AI, 5, 114. https://doi.org/10.3389/frobt.2018.00114

Orben, A. (2020). The Sisyphean Cycle of Technology Panics. Perspectives on Psychological Science, 15(5), 1143-1157. https://doi.org/10.1177/1745691620919372 
Panova, T., \& Carbonell, X. (2018). Is smartphone addiction really an addiction? Journal of Behavioral Addictions, 7(2), 252-259. https://doi.org/10.1556/2006.7.2018.49

Sherman, L. E., Michikyan, M., \& Greenfield, P. M. (2013). The effects of text, audio, video, and in-person communication on bonding between friends. Cyberpsychology: Journal of Psychosocial Research on Cyberspace, 7(2). https://doi.org/10.5817/CP2013-2-3

Short, J., Williams, E., \& Christie, B. (1976). The social psychology of telecommunications. Toronto; London; New York: Wiley.

Sun, J., Harris, K., \& Vazire, S. (2020). Is well-being associated with the quantity and quality of social interactions? Journal of Personality and Social Psychology, 119(6), 1478-1496. https://doi.org/10.1037/pspp0000272

Tamir, M., John, O. P., Srivastava, S., \& Gross, J. J. (2007). Implicit theories of emotion: Affective and social outcomes across a major life transition. Journal of Personality and Social Psychology, 92(4), 731-744. https://doi.org/10.1037/0022-3514.92.4.731

Twenge, J. M., Martin, G. N., \& Campbell, W. K. (2018). Decreases in psychological well-being among American adolescents after 2012 and links to screen time during the rise of smartphone technology. Emotion, 18(6), 765-780. https://doi.org/10.1037/emo0000403

Verduyn, P., Schulte-Strathaus, J. C. C., Kross, E., \& Hülsheger, U. R. (2021). When do smartphones displace face-to-face interactions and what to do about it? Computers in Human Behavior, 114, 106550. https://doi.org/10.1016/j.chb.2020.106550

Won, A. S., Shriram, K., \& Tamir, D. I. (2018). Social Distance Increases Perceived Physical Distance. Social Psychological and Personality Science, 9(3), 372-380. https://doi.org/10.1177/1948550617707017 
Wood, A., Lipson, J., Zhao, F., \& Niedenthal, P. (2020). Forms and Functions of Affective Synchrony [Preprint]. PsyArXiv. https://doi.org/10.31234/osf.io/z46nd 


\section{Table 1}

Means, Standard Deviations, Intraclass Correlations, and Within-Person Correlations for Key Study Variables

\begin{tabular}{|c|c|c|c|c|c|c|c|c|c|c|c|c|c|}
\hline Variable & $M(S D)$ & ICC & 1 & 2 & 3 & 4 & 5 & 6 & 7 & 8 & 9 & 10 & 11 \\
\hline 1. Lonely & $3.52(1.64)$ & .54 & - & & & & & & & & & & \\
\hline 2. Sad & $3.41(1.58)$ & .47 & $.56^{* * *}$ & - & & & & & & & & & \\
\hline 3. Anxious & $4.00(1.69)$ & .44 & $.36^{* * * *}$ & $.55^{* * * *}$ & - & & & & & & & & \\
\hline 4. Affectionate & $4.20(1.58)$ & .48 & $-.50^{* * * *}$ & $-.33^{* * *}$ & $-.13^{*}$ & - & & & & & & & \\
\hline 5. Understood & $4.03(1.52)$ & .52 & $-.42^{* * * *}$ & $-.31^{* * * *}$ & $-.20^{* *}$ & $.55^{* * * *}$ & - & & & & & & \\
\hline 6. Happy & $4.36(1.36)$ & .36 & $-.54^{* * * *}$ & $-.63^{* * * *}$ &.$- .43^{* * * *}$ & $.56^{* * * *}$ & $.50^{* * * *}$ & - & & & & & \\
\hline 7. Mode & $.70(.995)$ & .16 & $.20^{* * *}$ & $.20^{* * *}$ & $.12^{*}$ & $-.23^{* * *}$ & $-.19^{* * *}$ & $-.23^{* * * *}$ & - & & & & \\
\hline 8. Length & $61.1(56.2)$ & .16 & -.10 & -.07 & -.06 & $.16^{* * * *}$ & $.14^{* *}$ & $.13^{* *}$ & $-.28^{* * * *}$ & - & & & \\
\hline 9. Initiated & $.53(.50)$ & .04 & $-.12^{* *}$ & -.06 & -.02 & $.14^{* *}$ & $.11^{*}$ & $.12^{* *}$ & -.02 & .02 & - & & \\
\hline 10. Positivity & $2.61(.56)$ & .12 & $-.29^{* * * *}$ & $-.27^{* * * *}$ & $-.14^{* *}$ & $.31^{* * * *}$ & $.34^{* * *}$ & $.42^{* * * *}$ & $-.29^{* * * *}$ & $.18^{* * *}$ & $.21^{* * * *}$ & - & \\
\hline 11. Meaning & $2.34(.65)$ & .09 & $-.25^{* * *}$ & $-.19^{* * * *}$ & -.09 & $.34^{* * * *}$ & $.28^{* * *}$ & $.28^{* * *}$ & $-.21^{* *}$ & $.27^{* * * *}$ & $.23^{* * *}$ & $.44^{* * *}$ & - \\
\hline 12. Connection & $2.40(.64)$ & .11 & $-.25^{* * * *}$ & $-.266^{* * * *}$ & -.08 & $.40^{* * * *}$ & $.34^{* * * *}$ & $.34^{* * * *}$ & $-.18^{* * * *}$ & $.17^{* *}$ & $.19^{* * *}$ & $.49^{* *}$ & $.63^{* * * *}$ \\
\hline
\end{tabular}

Note. Variables $1-6$ are reported on a scale of $1=$ "not at all" to $7=$ "very much". Mode $=$ interaction mode $(0=$ in-person, $1=$ video, 2 = phone, 3 = text); Length = length of the interaction in minutes (observed range in the sample: $2-240$ ); Initiated = whether or not the interaction was initiated by the participant $(0=$ no, $1=$ yes $)$; Variables $10-12$ are reported on a scale of $1=$ "not at all" to $3=$ "very".

${ }^{*} p<.05 .{ }^{* *} p<.01 .{ }^{* * *} p<.001$. 


\section{Figure 1}

Momentary Affective Experiences Following In-Person, Video-Based, Phone-Based, and Text-Based Interactions.

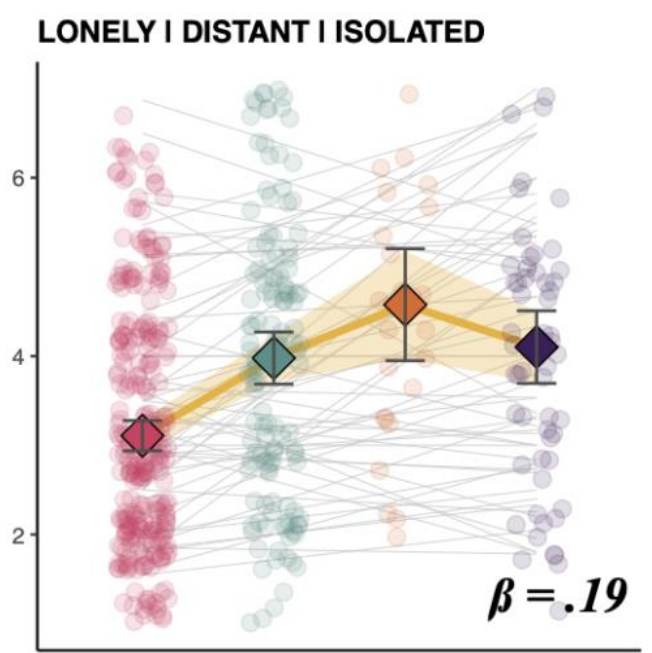

$\diamond$ In-person $\diamond$ Video $\diamond$ Phone $>$ Text
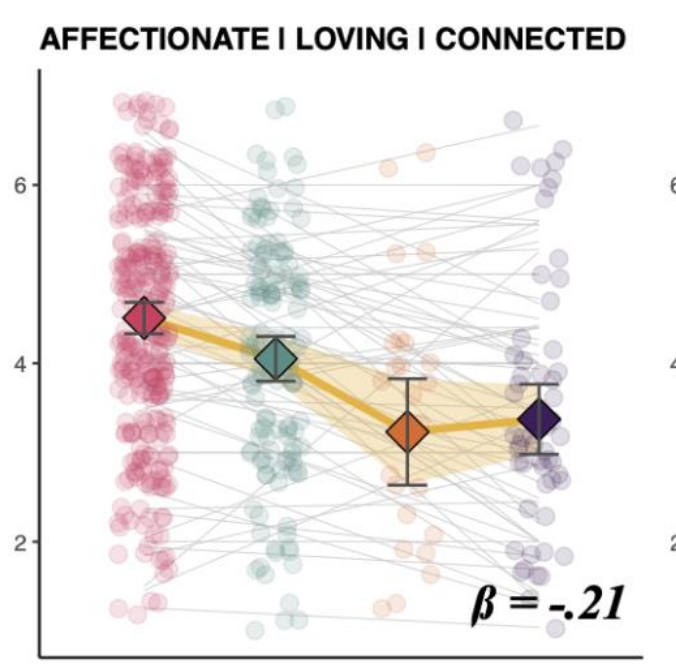

In-person $_{\text {Video }}>$ Phone $>$ Text

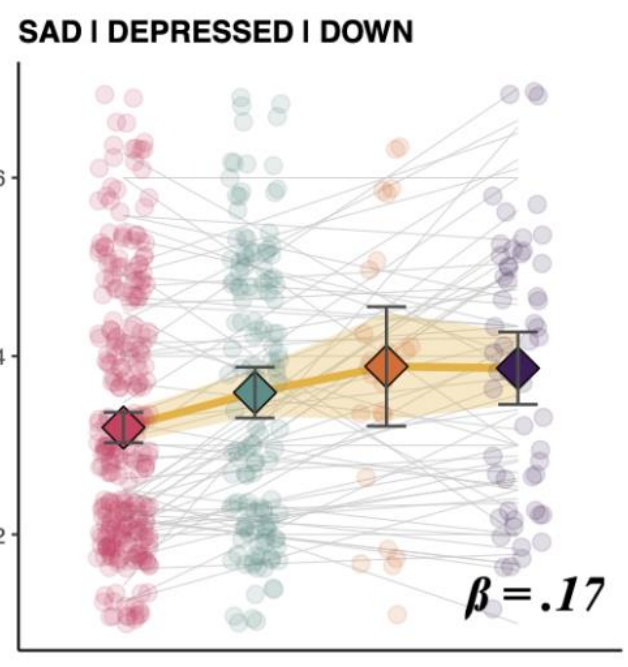

$\checkmark$ In-person $>$ Video $>$ Phone $>$ Text
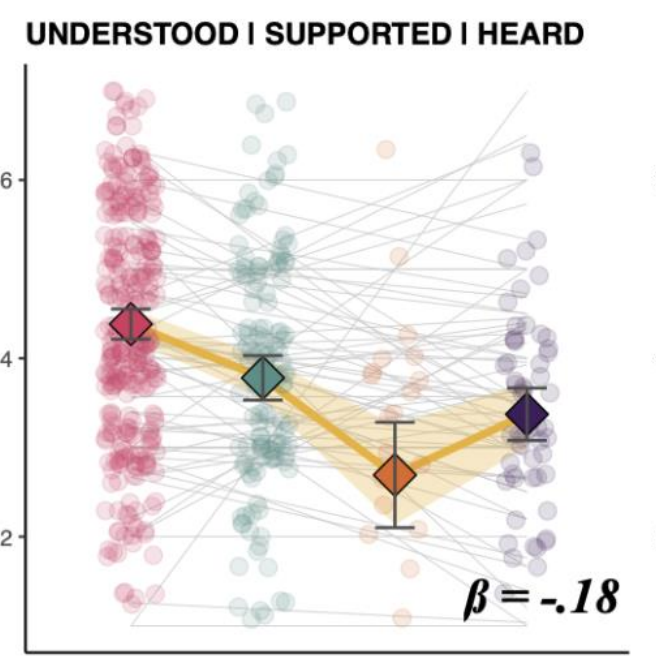

$>$ In-person $>$ Video $\diamond$ Phone $>$ Text
ANXIOUS I WORRIED I FEARFUL

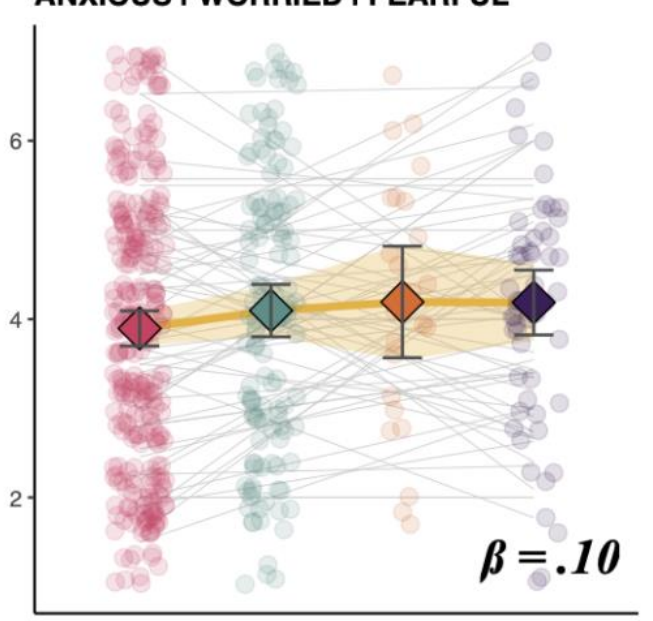

$\diamond$ In-person $\diamond$ Video $\diamond$ Phone $>$ Text

HAPPY I PLEASED I CONTENTED

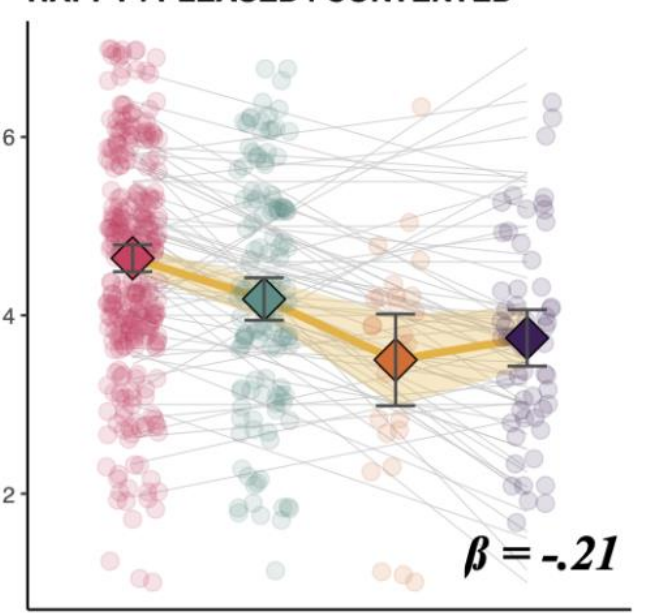

$\left.\nu_{\text {In-person }}\right\rangle$ Video $\diamond$ Phone $>$ Text 


\section{Figure captions}

Figure 1. Each round dot represents one interaction. The dots are semi-transparent, so that areas with darker colors indicate higher density of overlapping data points. Each diamond represents the average emotion intensity across all interactions of each type. Error bars represent $95 \%$ bootstrap CIs. Thin gray lines in the background represent estimated linear associations between interaction "life-likeness" and emotion intensity for each of the 102 participants over the course of 7 days (each gray line corresponds to one participant). Standardized (level-1) betas indexing the overall linear association between life-likeness and emotion reports are shown in the bottom right corner. 